\title{
THE COMPUTATION OF SURGERY GROUPS OF ODD TORSION GROUPS
}

\author{
BY ANTHONY BAK
}

Communicated by William Browder, February 24, 1974

The purpose of this note is to describe some of the main results pertaining to the computation of surgery groups of finite groups found in a joint paper [7] with W. Scharlau, one book [1] and five papers [2]-[6] of the author. Mention of the results is made in [10]. I shall indicate the source of each result. Throughout this note $\pi$ denotes a group. Let us begin with the results for surgery groups of odd torsion groups. Results closely related to the first three theorems have been announced in Wall [12].

THEOREM 1 [2]. If $\pi$ is an odd torsion group then the surgery obstruction groups

$$
L_{2 n+1}^{s, h}(\pi)=0 .
$$

Let $r_{\infty}$ denote the number (infinite if $\pi$ is infinite) of irreducible real representations of $\pi$.

THEOREM 2 [3]. If $\pi$ is an odd torsion group then the surgery obstruction groups

$$
\begin{aligned}
L_{2 n}^{s}(\pi) & =Z^{r_{\infty}} & & \text { if } n \equiv 0 \bmod 2, \\
& =Z^{r_{\infty}-1} \oplus Z_{2} & & \text { if } n \equiv 1 \bmod 2,
\end{aligned}
$$

and in the latter case the nontrivial element of $Z_{2}$ is represented by the based quadratic form $\left(Z \pi \oplus Z \pi,\left(\begin{array}{ll}1 & 0 \\ 1 & 1\end{array}\right)\right)$.

Let $Z \pi$ and $Q \pi$ be the integral and rational group rings of $\pi$. Let $K_{0}(Z \pi, Q \pi)$ be the relative group in the exact sequence of a localization [9, IX, §6] and let $\widetilde{K}_{0}(Z \pi)=K_{0}(Z \pi) /[Z \pi]$ be the projective class group of $Z \pi . K_{0}(Z \pi, Q \pi)$ is generated by pairs $(M, N)$ of finitely-generated projective $Z \pi$-lattices on a free $Q \pi$-module and if $M^{*}=\operatorname{Hom}_{Z \pi}(M, Z \pi)$, then $K_{0}(Z \pi, Q \pi)$ has a $Z_{2}$-action defined by $(M, N) \mapsto-\left(M^{*}, N^{*}\right)$, and $\tilde{K}_{0}(Z \pi)$ a $Z_{2}$-action defined by $M \mapsto-M^{*}$. Let $H^{0}\left(K_{0}(Z \pi, Q \pi)\right)$ be the zeroth cohomology group of the $Z_{2}$-action on $K_{0}(Z \pi, Q \pi)$ and let

$$
\boldsymbol{H}(\pi)=\text { coker } H^{0}\left(K_{0}(Z \pi, Q \pi)\right) \rightarrow \widetilde{K}_{0}(Z \pi) .
$$

AMS (MOS) subject classifications (1970). Primary 57-02, 57AXX, 57D65, 18-02, 18F25, 10C05, 16A54, 16-02 16A18, 16A26, 20C10, 20H99; Secondary 13D15. 
In [6] I show that when $\pi$ is finite abelian, $K_{0}(Z \pi, Q \pi) \cong I(Z \pi)$ (=group of invertible fractional $Z \pi$-ideals $), \tilde{K}_{0}(Z \pi) \cong \mathrm{Cl}(Z \pi)(=I(Z \pi) /$ principal fractional ideals), and the $Z_{2}$-actions on $K_{0}(Z \pi, Q \pi)$ and $\widetilde{K}_{0}(Z \pi)$ correspond to the natural involutions on $I(Z \pi)$ and $\mathrm{Cl}(Z \pi)$ given by the involution on $\boldsymbol{Q} \pi$.

THEOREM 3 [3]. If $\pi$ is an odd torsion group then

$$
\begin{aligned}
L_{2 n}^{h}(\pi) & =Z^{r_{\infty}} \oplus \boldsymbol{H}(\pi) & & \text { if } n \equiv 0 \bmod 2, \\
& =Z^{r_{\infty}-1} \oplus Z_{2} \oplus \boldsymbol{H}(\pi) & & \text { if } n \equiv 1 \bmod 2 .
\end{aligned}
$$

The nontrivial element of $Z_{2}$ is represented by the quadratic form $(Z \pi \oplus$ $\left.Z \pi,\left(\begin{array}{ll}1 & 0 \\ 1\end{array}\right)\right)$ and the elements of $\boldsymbol{H}(\pi)$ correspond to the classes of hyperbolic modules $\boldsymbol{H}(M)$ such that $M$ is finitely-generated projective and $M \oplus M^{*}$ is free.

Let $A$ be a ring with involution and let $\lambda \in$ center $A$ such that $\lambda \bar{\lambda}=1$. Let $K U_{0}^{\lambda}(A)\left(=K Q_{0}^{\lambda}(A, \max )\right.$, see $\left.[1, \S 1 \mathrm{~B}]\right)$ be the Grothendieck group of nonsingular even $\lambda$-hermitian forms on finitely-generated projective $A$-modules and let $W_{0}(A)=K U_{0}(A) /$ hyperbolic modules.

THEOREM 4 [3]. Let

$F$ number field with involution,

$E$ fixed field of the involution,

$S$ ring of integers in $F$,

$\lambda \lambda \in S$ such that $\lambda \bar{\lambda}=1$,

$\pi$ finite odd order or finite abelian group.

Give $S \pi$ the involution which sends each element of $\pi$ to its inverse and agrees with the involution on $S$.

(i) Assume $F$ has nontrivial involution, $E$ has an undecomposed real prime, and $F / E$ is totally unramified. Then $W_{0}(S \pi)$ is torsion free of the same (finite) rank as $W_{0}(F \pi)$.

(ii) Assume $F$ has trivial involution, $F$ is real, and each rational prime 1 [ $\pi: 1]$ is inert. Assume either $\lambda=-1$ or the class number of $F$ is odd. Then $W_{0}(S \pi)$ is torsion free of the same (finite) rank as $W_{0}(F \pi)$.

Let $K Q_{0}^{\lambda}(A)\left(=K Q_{0}^{\lambda}(A, \min )\right.$, see $\left.[1, \S 1 \mathrm{~B}]\right)$ be the Grothendieck group of nonsingular quadratic forms on finitely-generated projective $A$-modules.

THEOREM 5 [3]. If $\pi$ is a finite odd order group then

$$
\begin{aligned}
& K U_{0}^{\lambda}(Z \pi)=Z^{r_{\infty}+1} \oplus \boldsymbol{H}(\pi) \quad \text { if } \lambda=1 \text {, }
\end{aligned}
$$

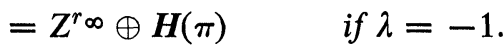

$$
\begin{aligned}
& K Q_{0}^{\lambda}(Z \pi)=Z^{r_{\infty+1}} \oplus \boldsymbol{H}(\pi) \quad \text { if } \lambda=1 \text {, }
\end{aligned}
$$

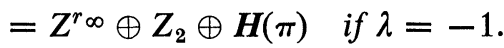


There is a general procedure described in $[1, \S \S 1 \mathrm{~B}, 3]$ to obtain results on Grothendieck groups of quadratic forms from Grothendieck groups of hermitian forms, and vise versa. We apply this now to the case of group rings. Let $K Q_{0}^{ \pm 1}(Z \pi)_{\text {based, proj }}$ (resp. $K U_{0}^{ \pm 1}(Z \pi)_{\text {based, proj }}$ ) be the Grothendieck groups of nonsingular quadratic (resp \pm 1 -hermitian) forms on finitely-generated based or projective $Z \pi$-modules. Let $W Q_{0}^{ \pm 1}(Z \pi)_{\text {based, proj }}$ (resp. $W_{0}^{ \pm 1}(Z \pi)_{\text {based, proj }}$ ) be $K Q_{0}^{ \pm 1}(Z \pi)_{\text {based, proj }}$ (resp. $\left.K U_{0}^{ \pm 1}(Z \pi)_{\text {based, proj }}\right)$ modulo hyperbolic modules on based or projective modules.

THEOREM 6 [1]. No assumption that $\pi$ be finite is made.

$$
\begin{aligned}
& K Q_{0}^{+1}(Z \pi)_{\text {based,proj }} \stackrel{\cong}{\longrightarrow} K U_{0}^{+1}(Z \pi)_{\text {based, proj }} \text {, } \\
& W Q_{0}^{+1}(Z \pi)_{\text {based,proj }} \stackrel{\cong}{\longrightarrow} W_{0}^{+1}(Z \pi)_{\text {based,proj. }} \text {. }
\end{aligned}
$$

(b) Assume that the elements of exponent 2 in $\pi$ generate a nilpotent subgroup (equivalently the 2-torsion elements generate a 2-group). Then the sequences below are split exact

$$
\begin{aligned}
& 0 \rightarrow Z_{2} \rightarrow K Q_{0}^{-1}(Z \pi)_{\text {based,proj }} \rightarrow K U_{0}^{-1}(Z \pi)_{\text {based,proj }} \rightarrow 0, \\
& 0 \rightarrow Z_{2} \rightarrow W Q_{0}^{-1}(Z \pi)_{\text {based,proj }} \rightarrow W_{0}^{-1}(Z \pi)_{\text {based,proj }} \rightarrow 0,
\end{aligned}
$$

and in both cases $Z_{2}$ is generated by the difference $\left[Z \pi \oplus Z \pi,\left(\begin{array}{ll}1 & 0 \\ 1 & 1\end{array}\right)\right]-$ $[\boldsymbol{H}(\mathrm{Z} \pi)]$.

Let $K Q_{1}^{ \pm 1}(Z \pi)$ (resp. $\left.K U_{1}^{ \pm 1}(Z \pi)\right)$ be $K_{1}$ of the category of nonsingular quadratic (resp. even \pm 1 -hermitian) forms on finitely-generated projective modules. For the standard matrix definition of these groups see $[1, \S \S 5,6]$. The next result has been obtained by Bass [8] in the case $\lambda=-1$ and by Siu [11] in the case $\lambda=1$ and $\pi$ cyclic.

THEOREM 7 [5]. Let $\pi$ be an odd torsion abelian group. Then there are split exact sequences

$$
\begin{aligned}
0 \rightarrow Z_{2} \rightarrow K Q_{1}^{1}(Z \pi)= & K U_{1}^{1}(Z \pi) \stackrel{\text { det }}{\longrightarrow} \pm \pi \rightarrow 0, \\
0 \rightarrow Z_{4} \rightarrow & K Q_{1}^{-1}(Z \pi) \stackrel{\text { det }}{\longrightarrow} \pi \rightarrow 0, \\
& K U_{1}^{-1}(Z \pi) \stackrel{\text { det }}{\cong} \pi .
\end{aligned}
$$

In the first case $Z_{2}$ is generated by the class of the matrix $\left(\begin{array}{rr}-1 & 0 \\ 0 & -1\end{array}\right)$ and in the second case $Z_{4}$ by $\left(\begin{array}{cc}0 & -1 \\ 1\end{array}\right)$.

For a related result in the nonabelian case see [2].

Give $\mathrm{Wh}(\pi)$ the involution defined by the conjugate transpose. The next result has been obtained also by Bass [8, §4]. 
THEOREM 8 [4]. If $\pi$ is a torsion abelian group then the involution on the Whitehead group $\mathrm{Wh}(\pi)$ is trivial.

Let $\hat{H}_{0}(\mathrm{Wh}(\pi))$ and $\hat{H}^{0}(\mathrm{Wh}(\pi))$ denote the reduced homology and cohomology groups of the involution on $\mathrm{Wh}(\pi)$. Let $r=$ number of irreducible rational representations of $\pi$ and let $r_{2}=Z_{2}$-rank $\left(Z_{2} \otimes \mathrm{Wh}(\pi)\right)$. The case $\pi$ abelian of the next theorem has been obtained also by Bass [8, §4].

THEOREM 9 [4]. (a) If $\pi$ is a finite odd torsion group then

$$
\hat{H}_{0}(\mathrm{Wh}(\pi))=0, \quad \hat{H}^{0}(\mathrm{Wh}(\pi))=Z_{2}^{r_{\infty}-r} .
$$

(b) If $\pi$ is a finite abelian group then

$$
\hat{H}_{0}(\mathrm{Wh}(\pi))=Z_{2}^{r_{2}}, \quad \hat{H}^{0}(\mathrm{Wh}(\pi))=Z_{2}^{r_{\infty}-r+r_{2}} .
$$

\section{BIBLIOGRAPHY}

1. A. Bak, K-theory of forms, Ann. Math. Studies (to appear).

2. - Odd dimension surgery groups of odd torsion groups vanish, Topology (to appear).

3. - The computation of even dimension surgery groups of odd torsion groups (preprint).

4. - - The involution on Whitehead torsion (preprint).

5. - Solution to the congruence subgroup problem for $\lambda$-hermitian forms (preprint).

6. - Grothendieck groups of modules and hermitian forms over commutative orders (preprint).

7. A. Bak and W. Scharlau, Grothendieck and Witt groups of orders and finite groups, Invent. fac. 1, 1974.

8. H. Bass, $L_{3}$ of finite abelian groups, Ann. of Math. (to appear).

9. - Algebraic K-theory, Benjamin, New York, 1968. MR 40 \#2736.

10. J. Shaneson, Hermitian K-theory in topology, Lecture Notes in Math., vol. 343, Springer-Verlag, Berlin and New York, 1973, pp. 1-40.

11. M. Siu, Computation of unitary Whitehead group of cyclic groups, Thesis, Columbia Univ., New York, 1971.

12. C. T. C. Wall, Some L groups of finite groups, Bull. Amer. Math. Soc. 79 (1973), $526-529$.

Department of Mathematics, Princeton University, Princeton, New Jersey 08540 .

Current address: Mathematisches Facultät, Universität Bielefeld, Bielefeld, Federal Republic of Germany 